\title{
Laser capture microdissection enables cellular and molecular studies of tooth root development
}

\begin{abstract}
Jian-Xun Sun ${ }^{1,2, *}$, Orapin V Horst ${ }^{3, *}$, Roger Bumgarner ${ }^{4}$, Bryce Lakely ${ }^{5}$, Martha J Somerman ${ }^{6}$ and Hai Zhang $^{7}$
Epithelial-mesenchymal interactions (EMIs) are critical for tooth development. Molecular mechanisms mediating these interactions in root formation is not well understood. Laser capture microdissection (LCM) and subsequent microarray analyses enable large scale in situ molecular and cellular studies of root formation but to date have been hindered by technical challenges of gaining intact histological sections of non-decalcified mineralized teeth or jaws with well-preserved RNA. Here, we describe a new method to overcome this obstacle that permits LCM of dental epithelia, adjacent mesenchyme, odontoblasts and cementoblasts from mouse incisors and molars during root development. Using this method, we obtained RNA samples of high quality and successfully performed microarray analyses. Robust differences in gene expression, as well as genes not previously associated with root formation, were identified. Comparison of gene expression data from microarray with real-time reverse transcriptase polymerase chain reaction (RT-PCR) supported our findings. These genes include known markers of dental epithelia, mesenchyme, cementoblasts and odontoblasts, as well as novel genes such as those in the fibulin family. In conclusion, our new approach in tissue preparation enables LCM collection of intact cells with well-preserved RNA allowing subsequent gene expression analyses using microarray and RT-PCR to define key regulators of tooth root development. International Journal of Oral Science (2012) 4, 7-13; doi:10.1038/ijos.2012.15; published online 16 March 2012
\end{abstract}

Keywords: gene; laser capture microdissection; microarray; PCR; root

\section{INTRODUCTION}

Tooth formation proceeds through a series of highly orchestrated reciprocal signaling interactions between dental epithelial and mesenchymal cells, termed epithelial-mesenchymal interactions (EMIs). ${ }^{1-5}$ Many genes/proteins involved in this process were identified and mapped temporally and spatially. ${ }^{6-7}$

In contrast to the crown formation, very few regulators of the root formation were identified. ${ }^{8-11}$ Work by our group and others suggests a role of epithelial signals in inducing the root development. The root forming cells such as cementoblasts (CB), dental follicle (DF) cells and other dental mesenchymal cells respond to enamel matrix proteins both in vitro and in vivo. ${ }^{1-18}$ Further support for a role of epithelial products in root development comes from tooth organ culture data and from investigation of genes expressed by Hertwig's epithelial root sheath. ${ }^{17,19-20}$ The data from these studies suggest that genes expressed by epithelial cells during root development are significantly different from those expressed during early stages of crown development; however, in depth studies to confirm these proposals, as well as knowledge as to specific similarities and/or differences in genes/proteins for crown vs. root development are minimal.

To determine the role of specific genes during tooth development, gene knockout animal models are often used. Many of these animal models are lethal and thus are only suitable for examining early stages of tooth development, i.e., crown development, since in rodents, first molar crown development is almost complete prior to birth. By limiting gene ablation to specific tissues and thus allowing for longer lifespans, conditional gene knockout models have served some purposes; however, without having specific markers for targeting tooth root development, such models have limited value. ${ }^{21-22}$

Laser capture microdissection (LCM) has been used extensively to analyze gene expression profiles in various non-mineralized tissues $^{23-24}$ and in the developing teeth at early stages of crown formation when the tooth is not highly mineralized. ${ }^{25-29}$ In mineralized tissue research, the difficulty in preparing intact unfixed, undecalcified and highly mineralized tissue sections (such as dentin and enamel) with wellpreserved cellular structures and high-quality RNA for LCM is a common obstacle. Over the past several years, we have optimized a technique for LCM of cells associated with the dento-alveolar bone complex. In this study, we aim to describe a new approach for tissue preparation and cell collection using LCM of postnatal day 7 (P7) and P14 mouse mandibles. This technique facilitates the use of LCM and microarrays to delineate regulatory mechanisms of the root development.

Mouse incisors are continuously erupting and have all stages of differentiating cells, while their molars develop in a similar fashion

${ }^{1}$ State Key Laboratory of Oral Diseases, Sichuan University, Chengdu, China; ${ }^{2}$ Department of Periodontics, University of Washington, Seattle, WA, USA; ${ }^{3}$ Division of Endodontics, Department of Preventive and Restorative Dental Sciences, University of California at San Francisco, San Francisco, CA, USA; ${ }^{4}$ Department of Microbiology, University of Washington, Seattle, WA, USA; ${ }^{5}$ Department of Urology, University of Washington, Seattle, WA, USA; ${ }^{6}$ National Institute of Arthritis and Musculoskeletal and Skin Diseases, National Institutes of Health, Bethesda, MD, USA and ${ }^{7}$ Department of Restorative Dentistry, University of Washington, Seattle, WA, USA

* These authors contributed equally to this work.

Correspondence: Dr H Zhang, 1959 NE Pacific St, Box 357456,Seattle, WA 98195, USA

E-mail: haizhang@u.washington.edu

Received 12 October 2011; Accepted 1 February 2012 
to humans. Therefore, we used both mouse incisors and molars in this study to define the regulatory mechanisms associated with the root formation and determine whether the EMIs are critical for the development of root tissues. ${ }^{30-32}$ This information should provide valuable insights and thus inform periodontal tissue regenerative therapy and tooth (crown and root) regeneration in the future.

\section{MATERIALS AND METHODS}

\section{Sample preparation}

As shown in Figure 1, lingual cervical loop (LCL) epithelial cells, adjacent apical dental papilla (DP) and apical DF cells were collected from mandibular incisors of three CD-1 mice on P7. Odontoblasts (OD) and $\mathrm{CB}$ were collected from roots of mandibular first molars from three mice on P14 when molar roots are actively developing. The protocol for animal use was approved by the University of Washington Institutional Animal Care and Use Committee. All equipment used for surgical dissection of mandibles was cleaned with RNaseZap (Applied Biosystem/ Ambion, Austin, TX, USA). After the erupted portion of mandibular incisors and excess bones were cut off (Figure 1a), the mandibles were embedded in an optimal cutting temperature (Tissue-Tek; Sakura
Finetek USA, Torrance, CA, USA) block using standard cryomolds (Sakura Finetek USA) and stored at $-80{ }^{\circ} \mathrm{C}$ until use.

For P7 incisor cervical loop epithelial and adjacent mesenchymal samples, the frozen mandibles were sectioned sagittally at a thickness of $14 \mu \mathrm{m}$ in a cryostat (CM3050 S; Leica Microsystems, Buffalo Grove, IL, USA) using a disposable tungsten carbide blade (TC-65; Leica Microsystems, Buffalo Grove, IL, USA). For P14 root OD and CB, the frozen mandibles were sectioned frontally at a thickness of $6 \mu \mathrm{m}$ at $-20{ }^{\circ} \mathrm{C}$. Six sections were placed per polyethylene naphthalate membrane frame slide (MDS Analytical Technologies, Sunnyvale, CA, USA) and kept at $-80{ }^{\circ} \mathrm{C}$ until staining and the laser capture microdissection were performed.

Section, staining, laser capture microdissection and RNA isolation Cell nuclei were stained with cresyl violet. Briefly, sections on each polyethylene naphthalate membrane slide were fixed with $95 \%$ ethanol for $1 \mathrm{~min}$, stained with $2.8 \%$ cresyl violet (MP Biomedicals, Solon, OH, USA) in 75\% ethanol, and dehydrated using the following series of alcohol and xylene: $95 \%$ ethanol for 1 min twice, $100 \%$ ethanol for $1 \mathrm{~min}$ twice and xylene for $5 \mathrm{~min}$ twice. The sections were

a

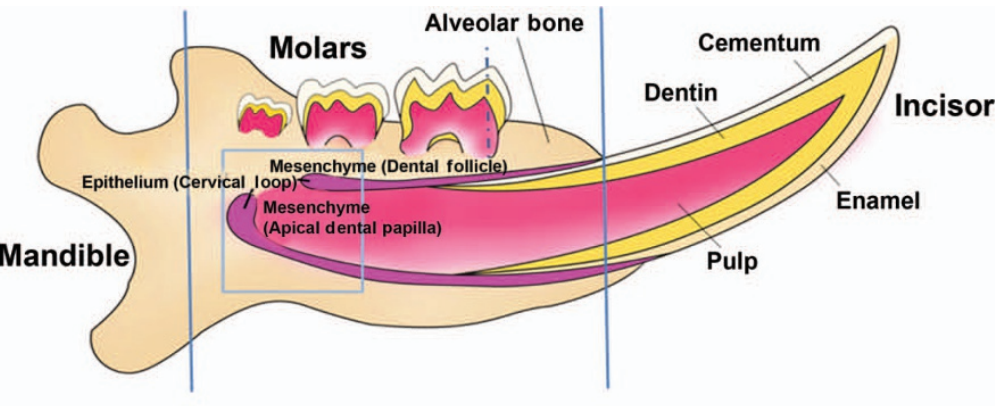

b

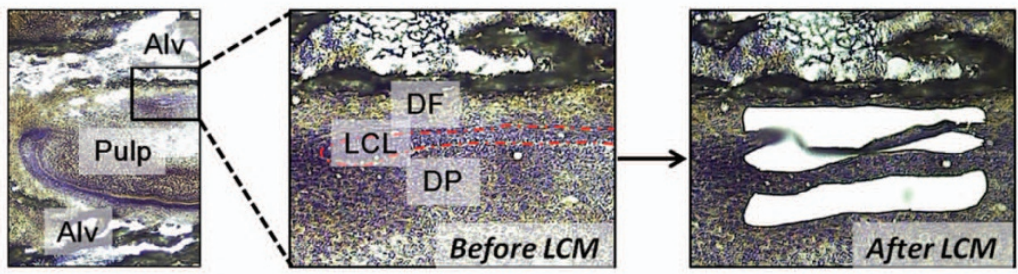

C

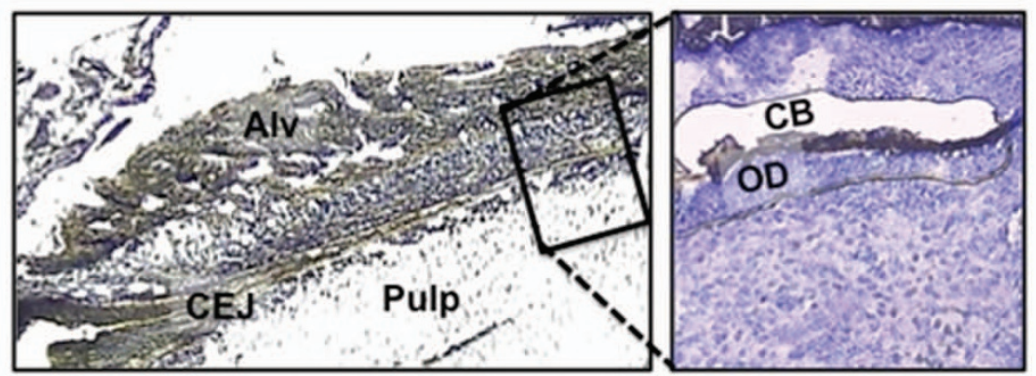

Figure 1 Laser capture microdissection (LCM) of dental epithelia, adjacent dental mesenchyme, odontoblasts and cementoblasts from sagittal sections of mouse mandibular incisors and molars. (a) Schematic illustration of a mouse mandible (sagittal plane) during tooth development. Rectangular frame shows the incisor cervical loop and surrounding area used for LCM (b). Dotted vertical line indicates frontal section of the first molar for LCM (c). Solid vertical lines indicate the orientation and size of the embedded tissues used in this study. Note the excess bones out of this border was trimmed off to make the sectioning more efficient and with high quality. (b) Undifferentiated epithelial and mesenchymal cells associated with incisor root formation. Cell nuclei are stained with cresyl violet (purple) on sagittal sections of the 7-day postnatal mouse mandible. The following three cell populations were captured: LCL (epithelia, red dotted line outlined), DP (mesenchyme) and DF (mesenchyme). Left panel: $\times 40$; middle panel: $\times 200$; right panel: $\times 200$. (c) Terminally differentiated cells associated with molar root formation, including: OD (dentin forming cells, not captured yet) and CB (cementum forming cells). Left panel: $\times 100$; right panel: $\times 200$. Alv, alveolar bone; CEJ, cemento-enamel junction; CB, cementoblasts; DF, dental follicle; DP, dental papilla; LCL, lingual cervical loop; OD, odontoblasts. 
allowed to dry at room temperature for $5 \mathrm{~min}$ and immediately used for laser capture microdissection using a Veritas Microdissection system (Arcturus Bioscience, Mountain View, CA, USA). Figure 1b and c provides a visual example to demonstrate the locations from which samples were taken. As CB and OD line the root surface as a single-cell layer, the underlying cementum and attached $\mathrm{CB}$, as well as the underlying dentin and attached OD, were dissected out together to minimize cellular damage caused by placing a laser beam directly onto the cells. The dissected tissues were collected onto the CapSure Macro LCM cap (Arcturus Bioscience). The collected tissue together with the membrane on the cap were detached from the cap and put into a $0.5 \mathrm{~mL}$ microcentrifuge tube containing $50 \mu \mathrm{L}$ of PicoPure lysis buffer and the tube was incubated at $42{ }^{\circ} \mathrm{C}$ for $30 \mathrm{~min}$, and processed for RNA isolation according to the manufacturer's instructions (Arcturus Bioscience). The RNA integrity number (RIN) was measured to evaluate the quality of each RNA sample, using an Agilent 2100 Bioanalyzer and RNA 6000 Pico Kit according to the manufacturer's instructions (Agilent Technologies, Santa Clara, CA, USA). The concentration of total RNA was quantified by UV absorption at $260 \mathrm{~nm}$ using a NanoDrop 1000 spectrophotometer (Thermo Fisher Scientific, Wilmington, DE, USA (Technical Support); Thermo Fisher Scientific, Waltham, MA, USA (Headquarters)).

\section{Gene expression microarray analysis}

Fifty nanograms of each total RNA sample were used for RNA amplification using Ovation Pico WTA System (NuGEN Technologies, San Carlos, CA, USA). Each amplified antisense cDNA product was converted into a sense cDNA transcript (ST-cDNA) and fragmented using WT-Ovation Exon Module (NuGEN Technologies). Each fragmented cDNA product was biotinylated, using an Encore Biotin Module according to the manufacturer's instructions (NuGEN Technologies).

Each biotinylated probe was hybridized onto a GeneChip Mouse Gene 1.0 ST Array (Affymetrix, Santa Clara, CA, USA) for $16 \mathrm{~h}$ at $45^{\circ} \mathrm{C}$. After hybridization, the array chips were washed, stained with streptavidin phycoerythrin using an Affymetrix Fluidics Station 450 and imaged using an Affymetrix Gene Chip Scanner 3000 (Affymetrix).

\section{Real-time reverse transcriptase polymerase chain reaction (RT-PCR)}

RT-PCR was used to verify mRNA expression of specific tissuerelated genes using the LightCycler 480 system (Roche Diagnostics, Mannheim, Germany). Twenty nanograms of each amplified cDNA sample were used. Primers were designed by the LightCycler probe design software (Roche Diagnostics, Indianapolis, IN, USA). Primer sequence analysis using a Basic Local Alignment Search Tool and melting curve analysis of the PCR products were used to ensure specificity of each primer pair. Primer sequences used in this study were as follows:

cytokeratin 14 (Krt14):

sense 5'-GCGGGATCAGTACGAG-3'

antisense 5'-AGTAACGGCCTTTGGT-3'

bone morphogenetic protein $2(\mathrm{Bmp} 2)$ :

sense 5'-CCCGATCACCTCTCTT-3'

antisense $5^{\prime}$-ACCGCAGTCCGTCTAA-3'

fibroblast growth factor 10 (Fgf10):

sense 5'-CCAGAGGGACCCTTAC-3'

antisense 5'-GGTTAAGCCCCAGGGA-3' bone sialoprotein (Bsp):

sense 5' - GAGACGGCGATAGTTCC-3'

antisense $5^{\prime}$ - AGTGCCGCTAACTCAA-3'

osteocalcin (Ocn):

sense 5' - TGAACAGACTCCGGCG-3'

antisense 5' - GATACCGTAGATGCGTTTG-3'

glyceraldehyde 3-phosphate dehydrogenase (Gapdh) as a house keep-

ing gene:

sense 5' $^{\prime}$ TCCACCACCCTGTTGCTGTA-3'

antisense 5'-ACCACAGTCCATGCCATCAC-3'

Using the LightCycler 480 software 1.5, the relative quantification of gene expression level for target vs. Gapdh (housekeeping gene) was performed (Roche). Amplification efficiency was determined for each pair of primers.

\section{Statistical analyses}

The robust multichip average (RMA) method was used for background adjustment, quantile normalization and median polish summarization using Affymetrix Expression Console software. Significance analysis of microarrays was used for statistical analyses of microarray data (http://www-stat.stanford.edu/ tibs/SAM/). Student's $t$-test was used for comparing the differential expression of each gene. $P<0.05$ was considered statistically significant.

\section{RESULTS}

\section{RNA integrity}

Using the protocol described in the 'Materials and methods' section, we were able to obtain a sufficient quality and quantity of RNA samples for global gene expression analyses using whole genome expression microarrays (Affymetrix mouse gene 1.0 ST). The RIN of each sample is shown in Table 1. RINs of all RNA samples were above or close to 6 . Instead of using traditional in vitro transcription from cDNA carrying the T7 promoter, we used Ribo-SPIA reactions with a combination of universal RNA primer, DNA polymerase and RNAse to isothermally amplify total RNA (Ovation Pico WTA System; NuGEN Technologies). Using this technique, we found that RNA samples with RIN above or close to 6 can be obtained and used successfully for whole genome microarray (Affymetrix mouse gene 1.0 ST).

\section{Quality of gene expression data from microarrays}

Data for quality control metrics of each array are shown in Table 2. Every array passed all quality control metrics. The area under the curve value for a receiver operating characteristic (ROC) curve plotted detection of positive controls against false detection of negative controls (Pos_vs_neg_auc) ranged from 0.84 to 0.89 for all arrays, indicating good array hybridization and signal quality for each gene expression microarray chip. Arrays were neither dim nor denoted as an outlier (Table 2 and Figure 2). After RMA expression, values were computed, and background correction and relatively identical distribution of expression values across arrays were ensured, indicating good array data quality.

Table 1 RNA integrity numbers

\begin{tabular}{lccccc}
\hline Samples & $\begin{array}{c}\text { Lingual } \\
\text { cervical } \\
\text { loop }\end{array}$ & $\begin{array}{c}\text { Apical } \\
\text { dental } \\
\text { follicle }\end{array}$ & $\begin{array}{c}\text { Apical dental } \\
\text { papilla }\end{array}$ & Odontoblasts & Cementoblasts \\
\hline 1 & 7.0 & 6.8 & 8.0 & 6.4 & 6.3 \\
2 & 7.0 & 8.5 & 7.1 & 6.4 & 6.5 \\
3 & 6.4 & 3.6 & 8.5 & 5.4 & 5.4 \\
\hline
\end{tabular}




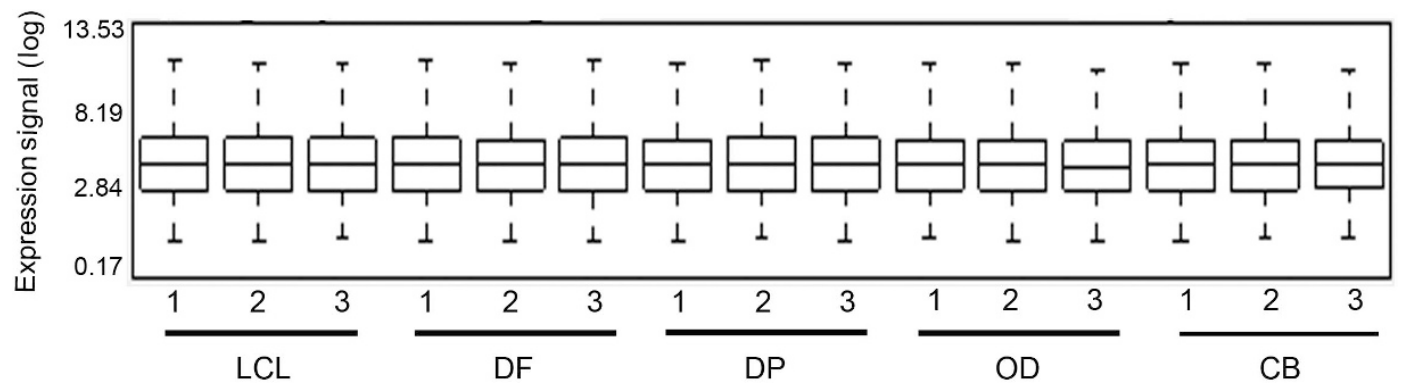

Figure 2 Box plots of log expression signals from all probes in each array after computing the RMA. Samples: LCL epithelium, apical DF and DP, OD and CB. All arrays had relatively similar distribution of expression values after RMA. No outliers were detected. CB, cementoblasts; DF, dental follicle; DP, dental papilla; LCL, lingual cervical loop; OD, odontoblasts; RMA, robust multichip average.

Robust differences in gene expression levels among tissues/cell types associated with the root formation

At $P<0.05$, significant differences in gene expression levels were found for 1710 genes between DP and LCL; 507 genes with differences greater than twofold (DP>LCL for 275 genes and LCL $>$ DP for 232 genes). Expression of 1265 genes differed significantly between DF and LCL; 327 genes with differences greater than twofold (DF>LCL for 188 genes and LCL $>$ DF for 139 genes). Expression of 1503 genes differed significantly between $\mathrm{OD}$ and $\mathrm{CB} ; 416$ genes with differences greater than twofold (OD>CB for 196 genes and $\mathrm{CB}>\mathrm{OD}$ for 220 genes) (Figure 3).

After adjusting for false discovery rate at $1 \%$ or less using significance analysis of microarrays, differential expression of 1014 genes among LCL, DF, DP, OD and CB was found to be significant at $P<0.05$. Two hundred and fifteen of these genes were expressed at higher levels in the DF and DP mesenchyme than in the LCL epithelia. These genes were also expressed at higher levels in the OD and CB than

Table 2 Quality metrics of gene expression microarray samples

\begin{tabular}{llccr}
\hline Samples & & Pos_vs_neg_auc $^{a}$ & PM_Mean $^{\text {b }}$ & Bgrd_Mean $^{c}$ \\
\hline Lingual cervical loop & 1 & 0.87 & 411.74 & 110.09 \\
& 2 & 0.85 & 412.57 & 121.19 \\
Apical dental follicle & 1 & 0.86 & 542.95 & 159.21 \\
& 2 & 0.87 & 402.88 & 114.89 \\
Apical dental papilla & 3 & 0.86 & 390.64 & 113.54 \\
& 1 & 0.88 & 464.59 & 135.82 \\
Root odontoblasts & 3 & 0.85 & 363.71 & 106.72 \\
& 3 & 0.86 & 358.40 & 99.52 \\
Root cementoblasts & 1 & 0.86 & 441.69 & 125.33 \\
& 2 & 0.86 & 250.25 & 75.45 \\
& 3 & 0.89 & 406.85 & 104.08 \\
& 3 & 0.86 & 234.75 & 78.08 \\
& 3 & 0.86 & 383.68 & 107.51 \\
& 0.84 & 212.35 & 71.63 \\
\hline
\end{tabular}

${ }^{a}$ Ratio for detection of positive controls (probes for exons of 100 housekeeping genes) vs. false detection of negative controls (probes for introns of the same 100 housekeeping genes). This metric indicates overall microarray data quality. Typical ratios range between 0.8 and 0.9 , with a value of 1.0 being perfect and a value of 0.5 showing no discernable difference between the positive and negative controls. A value significantly below 0.8 strongly indicates poor quality microarray data.

${ }^{b}$ Mean signal of all perfect match probes (oligonucleotide probes, typically 25-mers containing canonical sequence specific for each gene). These are raw signal intensities before normalization.

${ }^{c}$ Mean signal of background derived from antigenomic probes, which are designed not to hybridize to any genomic sequence in most studied organisms such as human, mouse, fly and etc. There are about 1000 probes for each level of GC content (0-25).
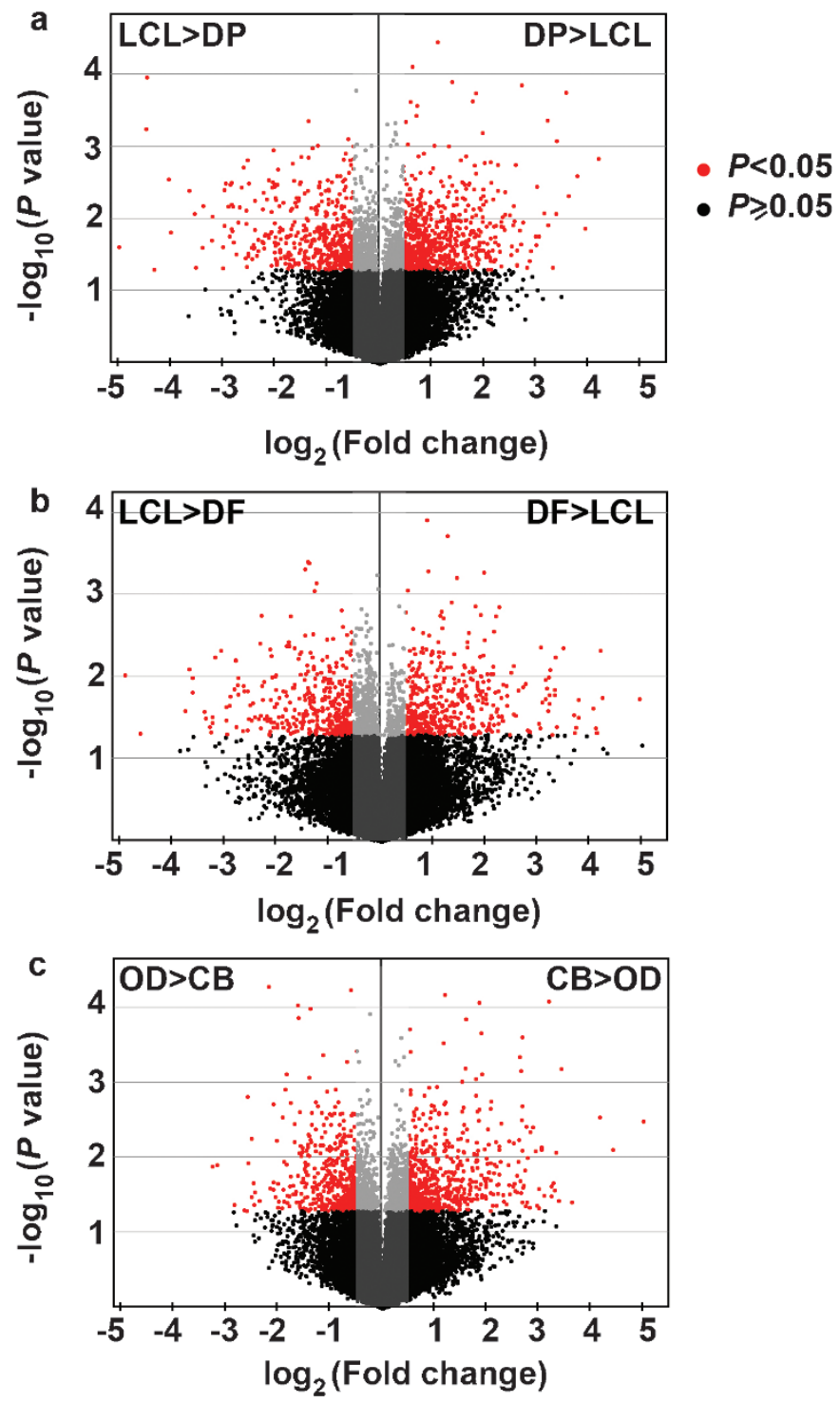

Figure 3 Volcano plots for differential expression of genes in LCL epithelia, adjacent DP mesenchyme, DF mesenchyme, root OD and CB. Unpaired $t$-tests were used to compare differences in gene expression. Genes with statistically significant difference at $P<0.05$ and FC (fold change) $\geqslant 2$ are shown in red, while those with $P \geqslant 0.05$ or $\mathrm{FC}<2$ are shown in grey or black (not statistically significant). CB, cementoblasts; DF, dental follicle; DP, dental papilla; LCL, lingual cervical loop; OD, odontoblasts. 

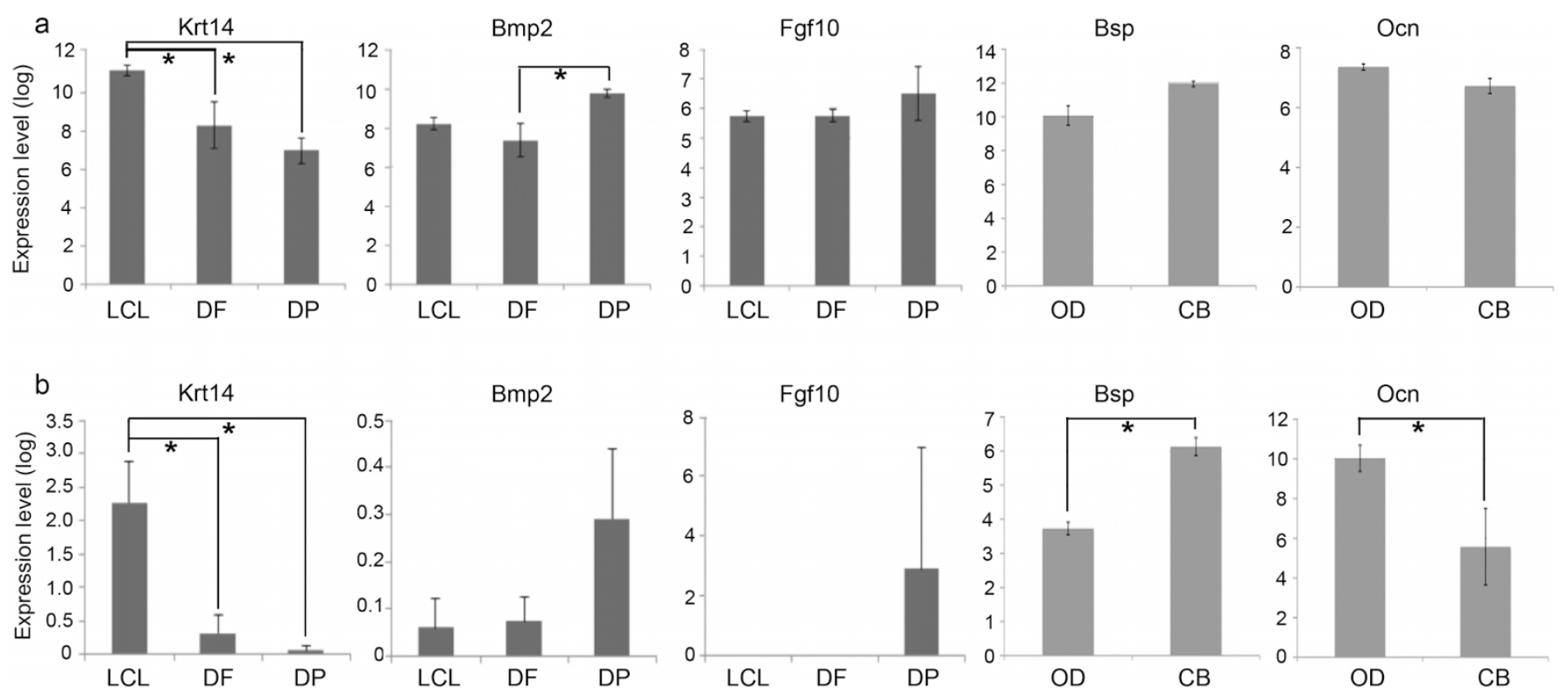

Figure 4 Comparison of mRNA expression data from microarray vs. RT-PCR. Genes known to be differentially expressed were selected and compared. mRNA expression levels derived from microarrays (a) were normalized by using the RMA method and those derived from quantitative RT-PCR (b) were normalized to the expression level of house keeping gene Gapdh as described in the 'Materials and methods' section. For LCL epithelium, Krt14; for dental mesenchyme including DF and DP, Bmp2 and Fgf10; for CB, Bsp; and for OD, Ocn. Differential expression patterns of these genes in LCL, DF, DP, CB and OD were generally consistent between microarray and RT-PCR data, though differences in measured gene expression levels between tissue/cell types were more robust in the data from RT-PCR than those from microarray. Bmp2, bone morphogenetic protein 2; Bsp, bone sialoprotein; Fgf10, fibroblast growth factor 10; Gapdh, glyceraldehyde 3-phosphate dehydrogenase; Krt14, cytokeratin 14; Ocn, osteocalcin; OD, odontoblasts; RMA, robust multichip average; RT-PCR, reverse transcriptase polymerase chain reaction.

in the DF and DP. In contrast, expression levels of 268 genes were greater in the LCL epithelial than in the mesenchymal cells including DF, DP, OD and CB. Seventeen genes were expressed at higher levels and more specifically in CB than in OD and other cells, while 26 genes were expressed higher and more specifically in OD than in $\mathrm{CB}$ and other cells.

Comparison of gene expression data from microarrays vs. RT-PCR Expression patterns of genes known to be selective to dental epithelium $v$ s. mesenchyme were examined to confirm specificity of cell isolation and to validate the microarray data. These genes include Krt14 for dental epithelium; Bmp2 and Fgf10 for early/undifferentiated dental mesenchyme (DF and DP); Ocn and Bsp for late/mineralized tissue forming mesenchyme (OD and $\mathrm{CB}$ ). Expression data of these genes derived from microarrays and from RT-PCR were compared (Figure 4). Krt14, an established epithelial cell marker, was expressed mainly in the LCL epithelium. In contrast, expression of Bmp2 and Fgf10 was greater in the adjacent DP than in the LCL and DF. Both OD and $\mathrm{CB}$ expressed $\mathrm{Bsp}$ and $\mathrm{Ocn}$; however, Bsp was expressed at higher levels in CB than in OD, while Ocn was expressed at higher levels in $\mathrm{OD}$ than in $\mathrm{CB}$, consistent with the previous analysis of these tissues by in situ hybridization. ${ }^{33}$

\section{DISCUSSION}

The techniques for LCM presented in this study were optimized to allow histological identification of odontogenic epithelia, mesenchyme and their lineage cells in the mineralized structures of teeth and mandibles while preserving the integrity of cellular component, particularly RNA. We demonstrated techniques for sectioning of unfixed and undecalcified frozen mouse mandibles, alcohol-based staining, LCM, RNA isolation, RNA amplification, microarray processing and subsequent gene expression data analyses. High-quality microarray data were noted, with high gene detection rates and signal-to-noise ratios (mean signals from perfect match probes $v s$. antigenomic probes/background) for all microarray chips processed. Further, high agreement rates were observed between gene expression data from microarrays and RT-PCR. These gene expression data also agreed with the limited findings from other studies using different techniques, showing success of our protocol presented here. ${ }^{13,34-38}$

Preservation of unfixed and undecalcified hard tissue samples during sectioning is challenging. Previous studies have shown a technique mounting these samples on transparent adhesive films to maintain tissue integrity. ${ }^{39-40}$ However, special equipment, materials and skills are required for this technique. Further, xylene cannot be used to completely dry the tooth tissues on the tapes. Using only a series of increasing concentration of alcohol to dry the tissues is frequently inadequate. The remaining water in the tissues increases the risk of RNA degradation from any residual/contaminated RNAses and also compromises the cutting ability of the laser beam during microdissection. By removing the unnecessary part of hard tissue, including the erupted part of mandibular incisors, surrounding mandibular bones and condyles, we showed that a conventional frozen section technique can be used to obtain sections of developing roots with well-preserved tissue integrity and histological structures.

Mammalian tissues are heterogenous, with several cell phenotypes and with various stages of differentiation within a given sample, presenting a challenge for in vivo molecular analyses. The LCM technique offers a superior way to collect target cells and minimize contamination with unwanted cells. During the LCM procedure, RNA degradation increases as processing time and procedures increase. ${ }^{41}$ RNAlater, an RNA stabilization reagent, was shown to improve RNA quality from the LCM samples. ${ }^{42-43}$ Using alcohol-based cresyl violet staining 
on frozen sections instead of aqueous stains such as hematoxylin also helps preserve RNA integrity, thereby improving the subsequent gene expression analyses using RT-PCR and microarrays. The removal of water rinsing steps and the use of higher concentrations of ethanol in our modified protocol minimize endogenous or exogenous RNase activity, thereby reducing RNA degradation. Similar findings with the alcohol-based staining method were also reported for laser capture microdissection of endometrial cancer, colon and brain tissues. ${ }^{41,44-45}$ In the present study, we found that the use of alcohol-based cresyl violet staining without RNAlater application was adequate. However, the RNAlater application may be useful for routine collection of clinical samples and requires further investigation.

Importantly, our findings of known genes at both gene expression level and tissue distribution are parallel to those described in the previous studies. Krt14 is highly expressed by odontogenic epithelial cells including cervical loop epithelia. ${ }^{34}$ Consistently, we found that Krt14 was expressed significantly higher in the LCLs of mandibular incisors than the mesenchymal counterparts. By contrast, Bmp2 and Fgf10 were expressed at significantly higher level in the pulpal mesenchyme than in the cervical loop epithelia, similar to the previous reports. ${ }^{13,35-36}$ Data from both in vitro and in vivo studies suggest that BMP2 induces the differentiation of odontogenic mesenchymal cells toward an odontoblastic lineage $e^{46-49}$ and may facilitate root dentin formation. FGF10 was shown to stimulate the proliferation of cervical loop epithelial cells, ectopic ameloblast differentiation and enamel formation on the lingual surface of mandibular incisors in sprouty-2 and -4 knockout mice (Spry4 ${ }^{-1-}$; Spry2 $2^{+/-}$mice). ${ }^{35}$ Similar to our findings, other researchers have reported that BSP is expressed at much higher level at both protein and gene levels in CB than in OD and thus is a good marker for cementum formation. ${ }^{36-37} \mathrm{OCN}$ is found in bone and dentin, as well as in the cells forming these two tissues (i.e., osteoblasts and $\mathrm{OD}$ ). $\mathrm{OCN}$ is considered as a marker for bone maturation. ${ }^{38}$ Our microarray data are consistent with those from RT-PCR and previous findings suggesting that our LCM technique presented here can be used successfully and reliably for a global study of gene expression and molecular interactions between odontogenic epithelial and mesenchymal stem cells during tooth root formation. Moreover, the technique described here should prove valuable for defining the behaviors of specific cells types associated with other mineralized tissues.

Microarray analyses provide large scale gene expression data that usually require further processing. Through analyses of our microarray data, some novel genes were revealed that exhibited differential expression patterns during the root formation. An example was the fibulin gene family. Fibulins are a family of secreted glycoproteins related to basement membranes, elastic fibers and other matrices. ${ }^{50}$ We found that six of the seven known fibulin family members, i.e., fibulin-1, -2, -3 (also known as Efemp1, EGF-containing fibulin-like extracellular matrix protein-1), -5 (also known as DANCE, EVEC), -6 (also known as Hemicentin-1 or Hmcn1) and -7 (also known as TM14) were expressed differentially in our developing incisor and molar root samples (data not shown). Excitingly, our microarray results suggest that some of these fibulins are differentially expressed in OD and CB. Importantly, in previous studies researchers noted fibulin-1 and -2 were expressed prominently in areas undergoing epithelial-mesenchymal transitions, while the expression of fibulin2 is more restricted. ${ }^{51-52}$ Fibulin-3 may play a role in the formation of cartilage and bone. ${ }^{53}$ Mutations in fibulin-5 were associated with agerelated macular degeneration, ${ }^{54}$ while the relationship between fibulin-6 and age-related macular degeneration is still controversial. ${ }^{55-56}$
Fibulin-5, -6 and -7 were shown to play a role in the cell attachment. ${ }^{57-59}$ Our ongoing studies are now focused on mapping the expression of this family of genes during tooth root development by in situ hybridization and immunohistochemistry using sections obtained from mice at different stages of tooth root development.

\section{CONCLUSIONS}

We describe a method for tissue preparation, cell collection and gene expression analyses of developing teeth using laser capture microdissection and microarrays. Using the presented protocol, we successfully performed the gene expression analyses and revealed robust gene expression changes, as well as novel genes associated with tooth root formation in mouse molars and incisors.

\section{ACKNOWLEDGEMENTS}

The authors would like to thank Dr Robert L Vessella and Dr Colm Morrissey in the Department of Urology, University of Washington for their technical support of LCM; Dr Theo Bammler, Dr Michael Coon and Dr Dick Beyer in the Department of Environmental and Occupational Health Sciences, University of Washington for their technical assistance in microarray analyses; Dr Brian Foster for his critical comments. This work is supported by NIH grant no. DE15109 to Dr Martha Somerman and a grant from the State Key Laboratory of Oral Diseases in Chengdu, China to Dr Hai Zhang.

1 Duailibi MT, Duailibi SE, Young CS et al. Bioengineered teeth from cultured rat tooth bud cells. J Dent Res 2004; 83(7): 523-528.

2 Kettunen P, Loes S, Furmanek T et al. Coordination of trigeminal axon navigation and patterning with tooth organ formation: epithelial-mesenchymal interactions, and epithelial Wnt4 and Tgfbeta1 regulate semaphorin 3a expression in the dental mesenchyme. Development 2005; 132(2): 323-334.

3 Kettunen P, Spencer-Dene B, Furmanek $T$ et al. Fgfr2b mediated epithelialmesenchymal interactions coordinate tooth morphogenesis and dental trigeminal axon patterning. Mech Dev 2007; 124(11/12): 868-883.

4 Luan X, Ito Y, Diekwisch TG. Evolution and development of Hertwig's epithelial root sheath. Dev Dyn 2006; 235(5): 1167-1180.

5 Thesleff I, Tummers M. Tooth organogenesis and regeneration. (January 31, 2009), StemBook [ed]. The Stem Cell Research Community, StemBook, doi/10.3824/ stembook.1.37.1, http://www.stembook.org/node/551.

6 Matalova E, Fleischmannova J, Sharpe PT et al. Tooth agenesis: from molecular genetics to molecular dentistry. J Dent Res 2008; 87(7): 617-623.

7 Zhang YD, Chen Z, Song YQ et al. Making a tooth: growth factors, transcription factors, and stem cells. Cell Res 2005; 15(5): 301-316.

8 Bosshardt DD, Nanci A. Immunodetection of enamel- and cementum-related (bone) proteins at the enamel-free area and cervical portion of the tooth in rat molars. $J$ Bone Miner Res 1997; 12(3): 367-379.

9 Dangaria SJ, Ito $\mathrm{Y}$, Walker $\mathrm{C}$ et al. Extracellular matrix-mediated differentiation of periodontal progenitor cells. Differentiation 2009; 78(2/3): 79-90.

10 Huang X, Xu X, Bringas P Jr et al. Smad4-Shh-Nfic signaling cascade-mediated epithelial-mesenchymal interaction is crucial in regulating tooth root development. J Bone Miner Res 2010; 25(5): 1167-1178.

11 Tummers M, Thesleff I. The importance of signal pathway modulation in all aspects of tooth development. J Exp Zool B Mol Dev Evol 2009; 312B(4): 309-319.

12 Boabaid F, Gibson CW, KuehI MA et al. Leucine-rich amelogenin peptide: a candidate signaling molecule during cementogenesis. J Periodontol 2004; 75(8): 1126-1136.

13 Harada $\mathrm{H}$, Kettunen $\mathrm{P}$, Jung $\mathrm{HS}$ et al. Localization of putative stem cells in dental epithelium and their association with Notch and FGF signaling. J Cell Biol 1999; 147(1): 105-120.

14 Le Norcy E, Kwak SY, Wiedemann-Bidlack FB et al. Leucine-rich amelogenin peptides regulate mineralization in vitro. J Dent Res 2011; 90(9): 1091-7.

15 Thesleff I, Wang XP, Suomalainen M. Regulation of epithelial stem cells in tooth regeneration. C R Biol 2007; 330(6/7): 561-564.

16 Wen X, Cawthorn WP, Macdougald OA et al. The influence of Leucine-rich amelogenin peptide on MSC fate by inducing Wnt10b expression. Biomaterials 2011; 32(27): 6478-686.

17 Zeichner-David M, Oishi K, Su Z et al. Role of Hertwig's epithelial root sheath cells in tooth root development. Dev Dyn 2003; 228(4):651-663.

18 Zhang H, Tompkins K, Garrigues J et al. Full length amelogenin binds to cell surface LAMP-1 on tooth root/periodontium associated cells. Arch Oral Biol 2010; 55(6): $417-425$.

19 Huang X, Bringas P Jr, Slavkin $\mathrm{HC}$ et al. Fate of HERS during tooth root development. Dev Biol 2009; 334(1):22-30. 
20 Zeichner-David M. Regeneration of periodontal tissues: cementogenesis revisited. Periodontol 2000 2006; 41: 196-217.

21 Fong $\mathrm{H}$, Chu EY, Tompkins KA et al. Aberrant cementum phenotype associated with the hypophosphatemic hyp mouse. J Periodontol 2009; 80(8): 1348-1354.

22 Foster BL, Nagatomo KJ, Bamashmous SO et al. The progressive ankylosis protein regulates cementum apposition and extracellular matrix composition. Cells Tissues Organs 2011; 194(5): 382-405.

23 Jacquet R, Hillyer J, Landis WJ. Analysis of connective tissues by laser capture microdissection and reverse transcriptase-polymerase chain reaction. Anal Biochem 2005; 337(1): 22-34.

24 Kai N, Iwase K, Imai K et al. Altered gene expression in the subdivisions of the amygdala of Fyn-deficient mice as revealed by laser capture microdissection and mKIAA cDNA array analysis. Brain Res 2006; 1073-1074: 60-70.

25 Liu D, Yao S, Wise GE. Effect of interleukin-10 on gene expression of osteoclastogenic regulatory molecules in the rat dental follicle. Eur J Oral Sci 2006; 114(1): 42-49.

26 Nakamura Y, Nomura Y, Arai C et al. Laser capture microdissection of rat periodontal ligament for gene analysis. Biotech Histochem 2007; 82(6): 295-300.

27 Wise GE, Ding D, Yao S. Regulation of secretion of osteoprotegerin in rat dental follicle cells. Eur J Oral Sci 2004; 112(5): 439-444.

28 Yao S, Ring S, Henk WG et al. In vivo expression of RANKL in the rat dental follicle as determined by laser capture microdissection. Arch Oral Biol 2011; 49(6): 451456.

29 Yao S, Pan F, Wise GE. Chronological gene expression of parathyroid hormone-related protein (PTHrP) in the stellate reticulum of the rat: implications for tooth eruption. Arch Oral Biol 2007; 52(3): 228-232.

30 Fong HK, Foster BL, Popowics TE et al. The crowning achievement: getting to the root of the problem. J Dent Educ 2005; 69(5): 555-570.

31 Foster BL, Somerman MJ. Regenerating the periodontium: is there a magic formula? Orthod Craniofac Res 2005; 8(4): 285-291.

32 Foster BL, Popowics TE, Fong HK et al. Advances in defining regulators of cementum development and periodontal regeneration. Curr Top Dev Biol 2007; 78: 47-126.

33 D'Errico JA, MacNeil RL, Takata T et al. Expression of bone associated markers by tooth root lining cells, in situ and in vitro. Bone 1997; 20(2): 117-126.

34 Kawano S, Saito M, Handa K et al. Characterization of dental epithelial progenitor cells derived from cervical-loop epithelium in a rat lower incisor. J Dent Res 2004; 83(2): 129-133.

35 Klein OD, Lyons DB, Balooch G et al. An FGF signaling loop sustains the generation of differentiated progeny from stem cells in mouse incisors. Development 2008; 135(2): 377-385.

36 Yamashiro T, Tummers M, Thesleff I. Expression of bone morphogenetic proteins and Msx genes during root formation. J Dent Res 2003; 82(3): 172-176.

37 Macneil RL, Sheng N, Strayhorn C et al. Bone sialoprotein is localized to the root surface during cementogenesis. J Bone Miner Res 1994; 9(10): 1597-1606.

38 Lee NK, Sowa H, Hinoi E et al. Endocrine regulation of energy metabolism by the skeleton. Cell 2007; 130(3): 456-469.

39 Kawamoto T, Shimizu M. A method for preparing 2- to 50-micron-thick fresh-frozen sections of large samples and undecalcified hard tissues. Histochem Cell Biol 2000; 113(5): 331-339.

40 Meacock SC, Brandon DR, Brown CP et al. A novel technique for immunohistoperoxidase staining of unfixed whole joints of small animals. Histochem $J$ 1992; 24(2): 115-119.

41 Clement-Ziza M, Munnich A, Lyonnet S et al. Stabilization of RNA during laser capture microdissection by performing experiments under argon atmosphere or using ethanol as a solvent in staining solutions. Rna 2008; 14(12): 2698-2704.
42 Guo D, Catchpoole DR. Isolation of intact RNA following cryosection of archived frozen tissue. Biotechniques 2003; 34(1): 48-50.

43 Roos-van Groningen MC, Eikmans M, Baelde HJ et al. Improvement of extraction and processing of RNA from renal biopsies. Kidney Int 2004; 65(1): 97-105.

44 Cummings $\mathrm{M}$, McGinley CV, Wilkinson $\mathrm{N}$ et al. A robust RNA integrity-preserving staining protocol for laser capture microdissection of endometrial cancer tissue. Anal Biochem 2011; 416(1): 123-125.

45 Wang WZ, Oeschger FM, Lee $\mathrm{S}$ et al. High quality RNA from multiple brain regions simultaneously acquired by laser capture microdissection. BMC Mol Biol 2009; 10 69

46 Casagrande L, Demarco FF, Zhang Z et al. Dentin-derived BMP-2 and odontoblast differentiation. J Dent Res 2010; 89(6): 603-608.

47 Chen S, Gluhak-Heinrich J, Martinez M et al. Bone morphogenetic protein 2 mediates dentin sialophosphoprotein expression and odontoblast differentiation via NF-Y signaling. J Biol Chem 2008; 283(28): 19359-19370.

48 Nakashima M. Bone morphogenetic proteins in dentin regeneration for potential use in endodontic therapy. Cytokine Growth Factor Rev 2005; 16(3): 369-376.

49 Yang X, van der Kraan PM, Bian Z et al. Mineralized tissue formation by BMP2 transfected pulp stem cells. J Dent Res 2009; 88(11): 1020-1025.

50 de Vega S, Iwamoto T, Yamada Y. Fibulins: multiple roles in matrix structures and tissue functions. Cell Mol Life Sci 2009; 66(11/12): 1890-1902.

51 Zhang HY, Timpl R, Sasaki T et al. Fibulin-1 and fibulin-2 expression during organogenesis in the developing mouse embryo. Dev Dyn 1996; 205(3): 348-364.

52 Tsuda T, Wang H, Timpl R et al. Fibulin-2 expression marks transformed mesenchyma cells in developing cardiac valves, aortic arch vessels, and coronary vessels. Dev Dyn 2001; 222(1): 89-100.

53 Ehlermann J, Weber S, Pfisterer $\mathrm{P}$ et al. Cloning, expression and characterization of the murine Efemp1, a gene mutated in Doyne-Honeycomb retinal dystrophy. Gene Expr Patterns 2003; 3(4): 441-447.

54 Lotery AJ, Baas D, Ridley C et al. Reduced secretion of fibulin 5 in age-related macular degeneration and cutis laxa. Hum Mutat 2006; 27(6): 568-574.

55 Schultz DW, Klein ML, Humpert AJ et al. Analysis of the ARMD1 locus: evidence that a mutation in Hemicentin-1 is associated with age-related macular degeneration in a large family. Hum Mol Genet 2003; 12(24): 3315-3323.

56 Brión M, Sanchez-Salorio M, Cortón M et al. Genetic association study of age-related macular degeneration in the Spanish population. Acta Ophthalmol2011; 89(1): e12e22.

57 Williamson MR, Shuttleworth A, Canfield AE et al. The role of endothelial cell attachment to elastic fibre molecules in the enhancement of monolayer formation and retention, and the inhibition of smooth muscle cell recruitment. Biomaterials 2007; 28(35): 5307-5318.

58 Vogel BE, Hedgecock EM. Hemicentin, a conserved extracellular member of the immunoglobulin superfamily, organizes epithelial and other cell attachments into oriented line-shaped junctions. Development 2001; 128(6): 883-894.

59 de Vega S, Iwamoto T, Nakamura T et al. TM14 is a new member of the fibulin family (fibulin-7) that interacts with extracellular matrix molecules and is active for cell binding. J Biol Chem 2007; 282(42): 30878-30888.

This work is licensed under a Creative Commons Attribution-NonCommercial-NoDerivative Works 3.0 Unported License. To view a copy of this license, visit http:// creativecommons.org/licenses/by-nc-nd/3.0 